\title{
Gut-liver axis: PPIs, Enterococcus and promotion of alcoholic liver disease
}

...overgrowth of $E$. faecalis

... alone exacerbated ethanolinduced liver disease...
Gastric acid suppression with proton-pump inhibitors (PPIs) promotes the progression of alcoholic liver disease (ALD) in mice via the overgrowth of intestinal Enterococcus faecalis, according to a new study. Moreover, in a large cohort of patients with chronic alcohol abuse, PPI use was associated with an increased risk of ALD, with increased numbers of Enterococcus spp. observed in faecal samples.

PPIs are among the most commonly prescribed medications worldwide. However, these agents are not without controversy, with an increasing number of concerns about PPI-associated risks and complications. "There has been a substantial growth in the total PPI use," notes author Bernd Schnabl. "Gastric acid kills ingested microbes, and suppression of gastric acid secretion can change the composition of the intestinal microbiota." PPI use is also seen in those with chronic liver disease, which can be associated with altered gut microbiota, and led the researchers to examine the effects of gastric acid suppression in these patients in a study supported by the NIH and the NIAAA.

The researchers used mutant mice (Atp $4 a^{\mathrm{SI} / \mathrm{sI}}$ mice) that develop achlorhydria - that is, they lack gastric acid - and fed them ethanol. They found that lack of gastric acid exacerbated alcohol-induced liver disease, including higher levels of severe liver injury and hepatic steatosis in mutant mice than in wild-type controls. Moreover, mutant mice progressed from simple steatosis to alcoholic steatohepatitis and had fibrotic livers. Crucially, ethanol administration led to intestinal bacterial overgrowth and dysbiosis in both strains of mice, but at markedly higher levels in $A t p 4 a^{\mathrm{S} / \mathrm{SI}}$ mice, with an increased proportion of Enterococcus spp. in the gut microbiota. Similar trends in increased liver disease progression and increased levels of Enterococcus spp. in the absence of gastric acid secretion were observed in mouse models of NAFLD (Atp $4 a^{\mathrm{SI} / \mathrm{SI}}$ mice fed a high-fat diet). In another model of NAFLD (mice fed a cholinedeficient L-amino acid-defined diet), lack of gastric acid secretion exacerbated NASH.

Moving to pharmacological studies, the researchers observed the same trends. Mice given PPIs to increase gastric $\mathrm{pH}$ to that of $A t p 4 a^{\mathrm{sI} / S \mathrm{I}}$ mice developed more severe ethanolinduced liver injury, steatosis and fibrosis than mice that did not receive PPIs. Again, intestinal bacterial overgrowth and increased abundance of Enterococcus spp. were observed after ethanol administration alongside PPIs. Importantly, overgrowth of E. faecalis (administered to mice with repeated gavages) alone exacerbated ethanol-induced liver disease in the absence of gastric acid suppression, and even mild liver disease was induced by $E$. faecalis in control mice that did not receive ethanol. They found that E. faecalis caused inflammatory responses in the liver, mediated by
Toll-like receptor 2 on Kupffer cells, which led to increased IL- $1 \beta$ secretion and liver damage in the mice. Notably, mice treated with the IL- $1 \beta$ receptor antagonist anakinra were protected from E. faecalis-exacerbated ALD.

Finally, in a patient cohort with chronic alcohol abuse $(n=4,830)$, the 10 -year risk of a diagnosis of ALD was $20.7 \%$ for active PPI users, $16.1 \%$ for previous users and $12.4 \%$ for never users. Active PPI users had significantly higher risk of developing ALD than previous users (adjusted HR 1.37; 95\% CI 1.00-1.88) or neverusers (adjusted HR 1.52; 95\% CI 1.21-1.91). Moreover, markedly higher levels of Enterococcus spp. were noted in faecal samples from patients who abuse alcohol taking PPIs than in those who do not use PPIs.

Schnabl postulates that the recent rise in use of gastric acidsuppressing medications might have contributed to the increased incidence of chronic liver disease. "Although obesity and alcohol use predispose to acid reflux requiring antacid medication, many patients with chronic liver disease take gastric acid-suppressive medication without appropriate indication," he warns, adding that clinicians should consider withholding medications that suppress gastric acid unless there is a strong medical indication.

Katrina Ray

ORIGINAL ARTICLE Llorente, C. et al. Gastric acid suppression promotes alcoholic liver disease by inducing overgrowth of intestinal Enterococcus. Nat. Commun. 8, 837 (2017) FURTHER READING Malfertheiner, P. et al. Proton-pump inhibitors - understanding the complications and risks. Nat. Rev. Gastroenterol. Hepatol.http://dx.doi.org/10.1038/ nrgastro.2017.117 (2017) 